\title{
Liquefied Petroleum Gas (LPG) Bottling Process and Required Safety During Bottling: A Case Study
}

\author{
Mohammad Shimul Hossain*, Mohammad Golam Muntasir Shehab, Soumik Mahmood Leon, \\ Simanto Kumar Pal, Rafsanjani Rafi, Rashed Alom Emon, Sujon Kumar Gain, \\ Mohammad Shaheen Shah
}

Department of Petroleum and Mining Engineering, Jashore University of Science \& Technology, Jashore, Bangladesh

Email address:

shimulpme@gmail.com (M. S. Hossain)

${ }^{*}$ Corresponding author

\section{To cite this article:}

Mohammad Shimul Hossain, Mohammad Golam Muntasir Shehab, Soumik Mahmood Leon, Simanto Kumar Pal, Rafsanjani Rafi, Rashed Alom Emon, Sujon Kumar Gain, Mohammad Shaheen Shah. Liquefied Petroleum Gas (LPG) Bottling Process and Required Safety During Bottling: A Case Study. Petroleum Science and Engineering. Vol. 3, No. 1, 2019, pp. 5-9. doi: 10.11648/j.pse.20190301.12

Received: January 26, 2019; Accepted: March 13, 2019; Published: April 10, 2019

\begin{abstract}
Energy security is very important in the current world. Liquefied Petroleum Gas (LPG) is one of the significant energy sources. LPG can be produced from natural gas processing as well as crude oil refining. Now LPG is available in different sizes of cylinder. The bottling system of LPG in the cylinder is the main discussion topic of this paper. Safety requirement during bottling work is also explained in this research article. LPG cylinder can be filled in both automatically and manually. The automatic filling system is more secure and effective. A visible check of the cylinder is needed before refilling. Washing and drying are also conducted before filling if necessary. To avoid gas leakage from cylinder valve "O" ring must be checked before gas filling, if it is missing in valve must put it. After putting the cylinder body weight, modern machine carousel runs to fill the LP gas in the cylinder. The automatic carousel machine can fill several cylinders at a time. Cylinder checked by automatic weight checking scale to be ensured filling the stipulated value. After this to check the valve leak of LPG filled cylinder for security and safety. If all the process is positive, it is needed to put a safety cap on the cylinder valve and attach the thermo-sleeve for more safety. Then the filled cylinders are kept in the storage for distribution. The Personal Protection Equipment's (PPEs) is must to wear during filling the cylinder.
\end{abstract}

Keywords: Liquefied Petroleum Gas (LPG), Bottling Process, Carousel Machine, Hazards, Safety

\section{Introduction}

Liquefied Petroleum Gas (LPG or LP Gas) refers to a mix of gaseous hydrocarbon compounds, primarily propane and butane. The high flammable LPG is an odorless \& colorless substance. The non-toxic gas is heavier than air. LPG is made during natural gas processing and oil refining. LPG is separated from unprocessed natural gas using refrigeration. LPG is extracted from heated crude oil using a distillation tower. This LPG can be separated into its three primary parts: propane, butane, and isobutene [1-9]. LPG is a clean and efficient fuel that emits $20 \%$ less carbon dioxide (CO2) than heating oil, $50 \%$ less $\mathrm{CO} 2$ than coal and $70 \%$ less $\mathrm{CO} 2$ than wood. LPG is used for cooking, heating and generating electricity in an environmentally friendly process [6, 8-13].

The demand of LPG is increasing in every year in the whole world. The highest LPG importers are USA, EU as well as northeast Asia such as China, Japan, South Korea. On the other hand, the largest exporters are Middle East, West Africa and Norway [6, 9, 14]. China imported 7.1 million tons of LPG in 2014 (propane, butane and mixed) and 4.2 million tons in 2013. USA exported about 14 million tons of LPG in 2014. Arabian Gulf region exported highest percentages of LPG in 2013 [9, 14]. 


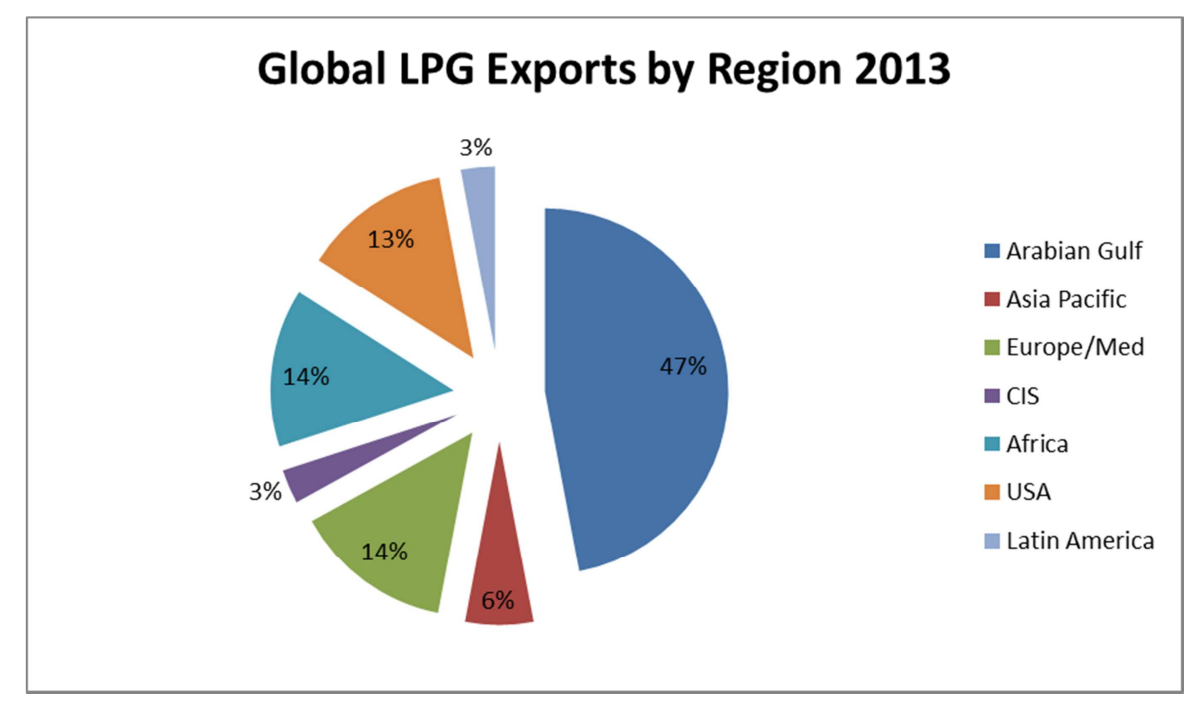

Figure 1. Global LPG Exports by Region 2013 [14].

The work was conducted on the LPG cylinder filling process and the safety during filling time. LP gas filled in different sizes of cylinders. Step by step of this process has been explained in this paper. Probable hazard and required safety during the work are also explained in this paper. Most of the LPG cylinders are made of steel. Plastic and aluminum are becoming popular alternatives to steel where weight is an issue. Composite type LPG cylinders are also interesting alternative to steel. The capacity of LPG Cylinders range from under $1 \mathrm{~kg}$ to $50 \mathrm{~kg}$ of $\mathrm{LPG}[1,4,7]$.

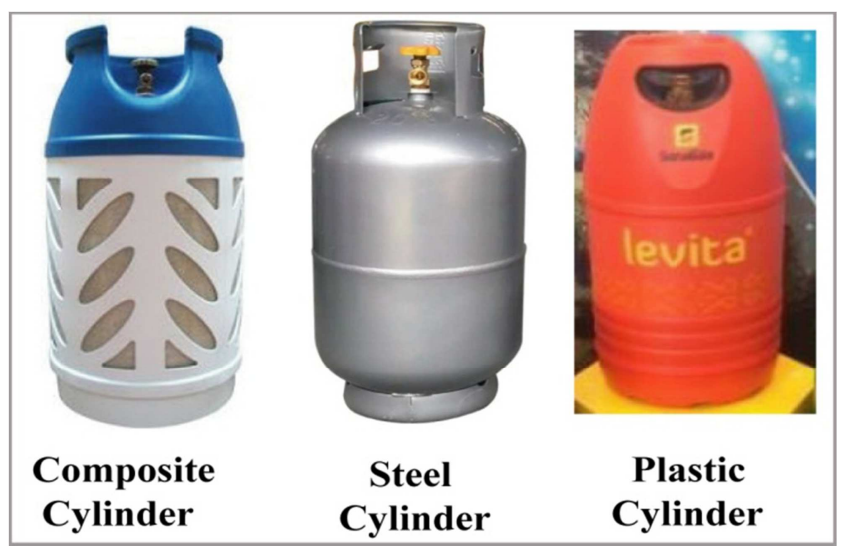

Figure 2. Variety of LPG Cylinders [1].

LPG, having high inflammability has fire and explosion hazards from production, distribution, transportation till been used and disposed [23]. Boiling Liquid Expanding Vapour Explosion (BLEVE) is the most destructive explosion hazard associated with LPG which can lead to highly destructive blast wave [24]. LPG may leak as a gas or a liquid. If it is leaked as liquid, it will expand to vapour by a factor or more than 200. Since LPG vapour is heavier than the air, it will settle down in the confined spaces and low-lying areas. Accumulation of LPG vapour may result in the development of an oxygen-deficient atmosphere which carries a risk of asphyxiation. When the gas meets a source of ignition it can burn or explode. Other hazards include lack of adequate ventilation is inadequate, Carbon monoxide produces from combustion may lead to narcotic effects, and LPG will cause cold burns if it comes into contact with the skin [23-25].

The poor quality control in LPG refining and production processes can lead to compromise in the safety and thus resulting in hazards during distribution and use. Thus, it is necessary to regulate and monitor the steps bottling process to minimize the potential risks during transport, distribution and finally utilization by the consumers because carelessness in any step might lead to hazard. Moreover, safety measures for the workers involved in the manufacture are crucial [2225].

\section{LPG Bottling Process}

Automatic bottling process is running in most of the LPG industry in the world. In all industries, there is also an alternative manual way to fill the cylinder. The manual filling process is needed when it faces problem in the automatic process. The automatically filling process is more secure and effective [15-18].

At first LPG cylinders are checked before filling for safety purpose. If it is needed to repair the cylinder then it is kept in the storage for future repair. If it is fit, the cylinder runs on the chain conveyor line for the filling process. Various online equipment is used for cylinder filling. The filling equipment is not exactly the same for all LPG industries but the following general steps for filling are followed by the industry [1, 15-18]. All the bottling processes are also shown by a flowchart in figure 4 .

\subsection{Washing Unit}

The Washing unit is that unit which is used to wash incoming cylinder. Multiple forced jet of water is used to remove mud, dirt, stains etc. from the outer surface of the cylinder. Most of the industries setup the on-line washing unit equipment. Some industries wash the outside of the cylinder by washing unit. There has a drying unit with 
washing unit to dry the cylinder after washing [1, 17-18]. It is needed to operate the unit by expert. When washing is needed, it could be run to wash the cylinder.

\section{2. “O” Ring Checking Unit}

Before filling the cylinder, "O" ring in cylinder valve should be checked. "O" is needed to remove LP gas leakage. After washing and drying, most of the industry put " $\mathrm{O}$ " ring in cylinder valve if the ring is missing there. Some industry put this after visible check $[1,18]$. It is very important to prevent gas leakage from cylinder.

\subsection{Tare Weight Unit}

Tare weight unit is the cylinder weight putting unit in a device. It is used to put the cylinder body weight so that the prescribed weight of LP gas can be filled accurately in the cylinder. All cylinders are not the same as body weight. So it is needed to set up the body weight of cylinder before filling. An operator put the tare weight by reading the body weight. Then transitional unit pushes the cylinder to carousel [19-20].

\subsection{Automatic Filling Carousel}

A filling carousel is a modern machine projected for filling LP gas cylinders in large groups. It contains a frame with running wheels, rails, a central column for LP gas and air, and a driving unit around the central column. The principal behind carousels is to use filling heads configured in a circular way where the empty cylinder enters the carousel, gets filled as it rotates around and then leaves the carousel filled at the point where it entered. The carousel can be fewer heads (e.g. eight) to larger heads (e.g. Seventy-two). The carousel can be filled in cylinder automatically. Every LPG industry has an alternative way to fill cylinder manually [19, 20].

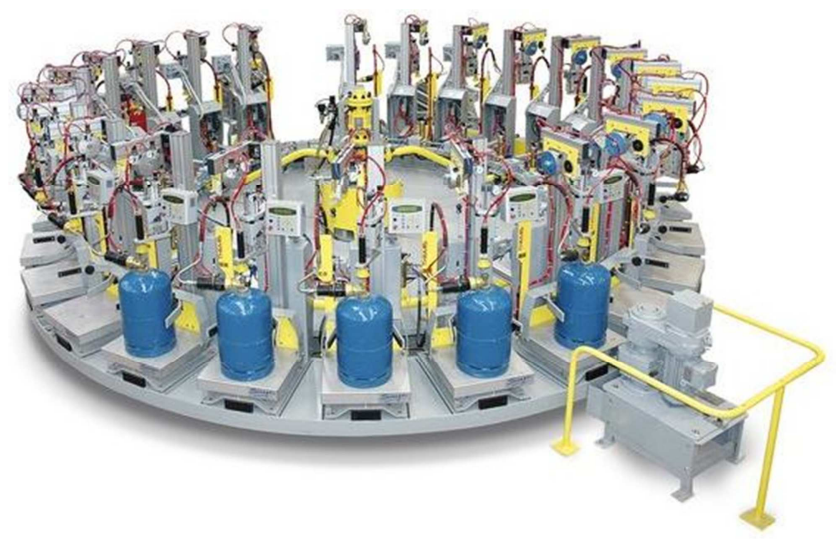

Figure 3. Cylinder Filling Carousel [20].

\subsection{Weight Checking Scale}

Weight checking scale or weighing scale is a device to measure weight. It is used for industrial and commercial application as well as for many other objects for sale. Weighing scale can be both manual or automatic in purpose. The scale can be various types as requirements.

Generally, Automatic weight checking scale is designed to check the gross weight of the filled LPG cylinder. Manual scales are also needed for any LPG industry. Rejected cylinder automatically to bypass line for filled properly manually if the gross weight of the cylinder more or less than the stipulated value. If the filled cylinder weight is good then it passed for leak detection [1, 17-18].

Washing Unit to wash cylinder outer surface, Check "O" ring in cylinder valve, Read cylinder body weight and set tare weight in Tabulation post

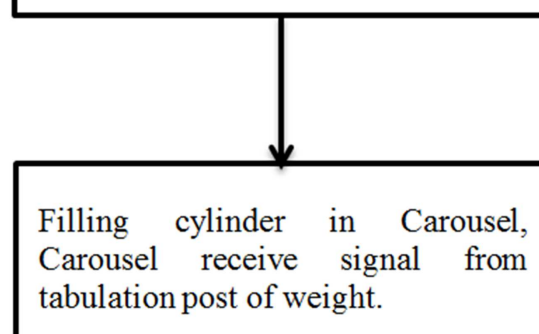

Put Safety Cap on valve and adjusted Thermo sleeve setting around the valve of LPG filled cylinder and then keep storage for distribution.

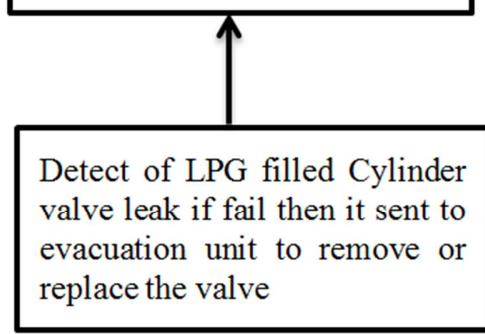

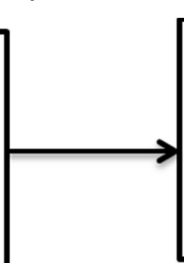

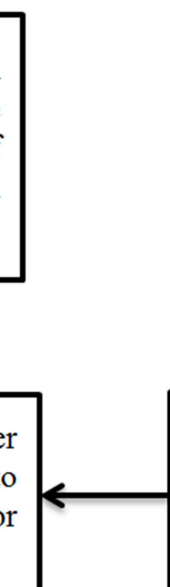

Carousel receive signal from tabulation post of weight. 


\subsection{Leak Detector Unit}

Leak detector is a device that is used in various industry to detect leak from an object. LPG industry uses it to detect LP gas filling cylinder leak. The leak can be of various types such as cylinder head leak, bottom leak, valve leak etc. A compact valve testing unit is on-line equipment designed to test cylinder valve leakage and rejects cylinder to bypass line for repairing if detect a leak. Remove or replace the leak valve of the cylinder by unscrewing or screwing at other places or on-line valve changing unit $[1,4]$.

Commonly, almost all LPG filling industry use automatic leak detector devise for checking valve and cylinder heal leak. Body leak is checked by soap water mostly manually.

\subsection{Safety Cap and Thermo-Sleeve Setting}

Safety cap is a protection cap that is used on cylinder valve. It is used for more safety to protect the gas leakage. After leak detection, safety cap fixes on the valve of LPG cylinder. Safety cap can be fixed manually in on-line running or can be used auto cap fixer on-line equipment. After capping, on-line equipment used to crimp aluminum cap seal/Thermo sleeve around the cylinder valve as a final operation and finishing safety of LPG cylinder filling [4].

After completing all the above process, the LPG filled cylinder keeps in storage for distribution to the customer.

\section{Probable Hazards and Required Safety During Bottling}

Safety is most important for any industry. Personal Protection Equipment (PPE) is used during working time for safety. In average, the hazards can be leakage, hand and leg injury, sound pollution, firing etc. Leakage of LPG from the cylinder can be occurred during filling in the carousel. LPG leakage can also be occurred during unscrewing/screwing. Hand and leg can be injured during lift up cylinder on-line chain conveyor. Sound pollution is also a problem during bottling [1, 4, 21-22].

To prevent hazards or getting injured during LPG cylinder filling, PPEs should be used. There are various types of PPEs such as safety shoe, hard hat (Helmet), safety glasses and gloves, reflective jacket, air muff, safety goggles etc. Safety shoes and gloves are always used to prevent leg and hand injury. Sound pollution can be minimized by using air muff. Face mask are always needed to use during the working time. Fire extinguishers are required to keep ready always for control any types of firing. $[1,4]$.

The initial check of the cylinder is needed to ensure that it is fit for filling. Cylinder valve is most important. Normally it is used for dual purpose, to refill and supply gas to the consumer. Filling a large number of cylinder at a time is very risky so it is safe to fill the cylinder automatically $[1,22]$.

\section{Conclusion and Suggestion}

Uses of LPG are safe and cheap. Use of LPG is getting popular day by day. Now, LPG is found in variety size cylinder due to customer demand. LPG filled in various types' cylinder such as steel cylinder, composite cylinder as well as the plastic cylinder. It can be filled both automatically and manually. The automatic filling process is more effective and can be filled more cylinder in a short time. For the safety, personal protection equipment should be used during whole working period. The succeeding recommendation should be followed during bottling process for more effective work and safety.

a. Always use required Personal Protection Equipment's (PPEs) while working in Bottling hall.

b. No smoking in bottling hall.

c. Safety shoes and gloves must be used all times.

d. Inspect each tool before use. Never use any defective cylinder.

e. It is needed to check initially whether the cylinder is fit for refilling.

f. Unauthorized persons should not be allowed in the working area.

g. Must go in Safety place when siren whistling.

\section{Acknowledgements}

The authors like to thank to the respectable reviewers for giving proper instruction to improve the quality of this paper. The authors also like to thank to Mir Raisul Islam for his cooperative help to improve the paper quality.

\section{References}

[1] Guide to Good Industry Practices LPG Cylinder Filling, 26 July 2017, World LPG Association, www.wlpga.org

[2] General Specification for Liquified Petroleum Gas Installation in Government Buildings of the Hong Kong special administrative region, 2012.

[3] Paczuski, M., Marchwiany, M., Puławski, R., Pankowski, A., Kurpiel, K. and Przedlacki, M., 2016, Liquefied Petroleum Gas (LPG) as a Fuel for Internal Combustion Engines, Alternative Fuels, Technical and Environmental Conditions, pp. 105-136.

[4] Guidelines for Good safety practices in the LP Gas Industry, 08 July 2008, World LPG Association, www.wlpga.org

[5] Bahadori, A., Liquefied Petroleum Gas (LPG) Recovery, 2014, Natural Gas Processing.

[6] World LP Gas Association Annual Report 2017.

[7] Guidelines for Good business practices in the LP Gas Industry, 08 July 2008, World LPG Association, www.wlpga.org 
[8] Cho. C. P., Kwon, O. S., Lee, Y. J., Effects of the sulfur content of liquefied petroleum gas on regulated and unregulated emissions from liquefied petroleum gas vehicle, 2014, Fuel, Vol. 137 pp. 328-334.

[9] Shah, M. S., Hossain. M. S., Nip, F. Y., Pa, S. K., Islam, M. R., Sany, S. M. S. Future Prospect Analysis of Liquefied Petroleum Gas (LPG) and Liquefied Natural Gas (LNG) in Bangladesh: Economic and Technological Case Studies, July 2018, Journal of Nature Science and Sustainable Technology, Volume 12, Issue 3.

[10] Elgas Ltd. (2016). http://www.elgas.com.au (Accessed on 25 September, 2016).

[11] Karim, M. (2016). Encouraging the use of LPG by households. Published: April 28, 2016 Thursday.

[12] Ahmad, R. M.; Majed, A.; Osama, E. D. H.; Marcos, S. F.; and Usman, M. A. Fractionation of Natural Gas Liquids to produce LPG. TPG4140 Natural Gas, Norway, 2011.

[13] Shipman MBE, R. H., MIMechE, MIGasE, MInstE, Liquefied petroleum gas, 2002, Plant engineer's Reference Book, Second Edition.

[14] Poten \& Partners. LPG in the world market, A Monthly Report on International Trends in LPG, February, 2015.

[15] ISO 22991 - Gas cylinders -Transportable Refillable Welded Steel Cylinders for Liquefied Petroleum Gas (LPG) - Design and Construction.
[16] ISO 10691 - Gas Cylinders - Refillable welded steel cylinders for liquefied petroleum gas (LPG) --Procedure for checking before, during and after filling.

[17] Kosan Crisplant (www.kosancrisplant.com)

[18] Siraga (www.siraga.com)

[19] Carrousel Filling Systems, 02 February 2017, Kosan Crisplant.

[20] LPG Filling Carrousel, 2018, Siraga. www.siraga.com

[21] UK's Health and Safety Executive.

[22] Guide to Gas Cylinder, 2013, Environmental Protection Authority, Technical Guide, New Zealand Government.

[23] World LPG Association (WLPGA), Guidelines for Good business practices in the LP Gas Industry, 2017.

[24] Oueidat D., Guarniere F., Gorbolino E. and Rigaud E., Evaluating the safety operations procedure of the LPG storage and distribution plant with STAMP, Elsevier Ltd., 2015

[25] Singh, S. and Premi, S. R., Hazard Identification and Risk Assessment in LPG Bottling Plant, International Journal of Science Technology \& Engineering, 1(12), pp 19-22, 2015. 